\title{
Expression of Aquaporin 1 and 5 in the Developing Mouse Inner Ear and Audiovestibular Assessment of an Aqp5 Null Mutant
}

\author{
Michele Merves, ${ }^{1}$ Carissa M. Krane, ${ }^{2}$ Hongwei Dou, ${ }^{1}$ John H. Greinwald, ${ }^{1}$ Anil G. Menon, ${ }^{3}$ \\ AND DANiEl CHOO ${ }^{1}$ \\ ${ }^{1}$ Department of Pediatric Otolaryngology, Center for Hearing and Deafness Research, Cincinnati, OH 45229, USA \\ ${ }^{2}$ Department of Biology, University of Dayton, Dayton, OH 45404, USA \\ ${ }^{3}$ Department of Molecular Genetics, Biochemistry and Microbiology, University of Cincinnati College of Medicine, Cincinnati, \\ OH 45229, USA
}

Received: 1 July 2002; Accepted: 7 November 2002; Online publication: 8 April 2003

\section{ABSTRACT}

To examine the potential roles of aquaporins 1 and 5 (AQP1 and AQP5, respectively) in inner ear development and function, we defined their spatial and temporal expression patterns in the developing mouse inner ear and examined the morphologic and physiologic effects of loss of Aqp5 function. Standard in situ hybridization (ISH) and immunohistochemical (IHC) assays were used for expression studies with routine morphologic, behavioral, and physiologic assessments of hearing and balance in Aqp5 null mutant mice. AQP1 was first detected at embryonic day 10.5 (E10.5) in the otocyst but eventually localized to specific nonsensory portions of the inner ear and connective tissue cells surrounding the membranous labyrinth. AQP5 displayed specific cochlear expression, first detectable at E15.5 in the nonsensory epithelium and later restricted to the lateral wall of the cochlear duct near the spiral prominence. AQP5 expression continued through postnatal periods with a change of expression domain to the stria vascularis between postnatal day 7 (P7) and P14. By in situ hybridization and immunohistochemical techniques, subtle differences between transcript and protein expression patterns were noted for both AQP1 and 5 . Although AQP5 is dynamically expressed in the de-

Correspondence to: Daniel Choo - Center for Hearing and Deafness Research • Department of Pediatric Otolaryngology • 3333 Burnet Avenue - Cincinnati, OH 45229. Telephone: (513) 636-4870; fax: (513) 636-2886; email: daniel.choo@cchmc.org veloping mouse inner ear, adult $A q p 5$ knockout mice show normal hearing when tested and normal inner ear structural development. These results suggest redundant or alternative mechanisms that likely regulate water homeostasis in the developing and mature inner ear.

Keywords: aquaporin, inner ear, development, endolymph, perilymph

\section{INTRODUCTION}

Development of the inner ear is a complex process that ultimately generates an intricate three-dimensional labyrinth that transduces both auditory and vestibular stimuli by means of a hair cell transduction mechanism (Corey and Hudspeth 1979; Hudspeth 1985; Morsli et al. 1998). Critical to this hair cellmediated process is a unique inner ear fluid environment with high potassium ion and low sodium ion concentrations and a net $+80 \mathrm{mV}$ potential relative to the surrounding compartments (Peake et al. 1969; Sadanaga and Morimitsu 1995). Data suggest that gated channels at the stereocilia of the cochlear and vestibular hair cells allow a flux of ions down this electrochemical gradient in response to sound or balance stimuli (Corey and Hudspeth 1979; Hudspeth 1985). Accordingly, mechanisms responsible for developing and maintaining this very specific fluid 
environment in the inner ear are essential to normal hearing and balance function.

Clinically, several human deafness and vestibular disorders (Paparella 1991; Everett et al. 1997; Karet et al. 1999; Kubisch et al. 1999) point toward a disruption of normal fluid homeostasis as a pathogenetic factor and implicate the importance of these fluid regulatory mechanisms to inner ear function. As an example, Meniere's disease is a process in which an overaccumulation of endolymph (endolymphatic hydrops) is thought to produce symptoms of fluctuating sensorineural hearing loss, episodic vertigo, tinnitus, and aural fullness (Kimura 1976; Paparella 1991). This theory on hydrops is in part based upon human temporal bone studies showing dilation of the endolymphatic compartment in inner ear specimens obtained from patients having been diagnosed with Meniere's disease prior to death (Schuknecht 1993). Further indirect evidence of a perturbation of inner ear fluid homeostasis comes from the varying success in alleviating Meniere's disease symptoms using medical therapies (e.g., diuretics) or surgical procedures (e.g., endolymphatic sac shunting) aimed at reducing endolymphatic hydrops and restoring normal endolymph homeostasis (Arenberg 1982; Welling et al. 1996; Shah and Kartush 1997; Sajjadi et al. 1998; Claes and Van de Heyning 2000). Therefore, the study of endolymph regulatory mechanisms has direct relevance to better understanding inner ear biology and potentially toward designing pharmacologic therapies to improve and/or restore normal endolymph homeostatic mechanisms.

To date, most studies of inner ear fluid (endolymph) homeostasis have focused on major cationic and anionic transport mechanisms (Naftalin and Harrison 1958; Juhn et al. 1976; Yamasaki et al. 2000; Steel and Kros 2001). However, with the identification of the aquaporin family of water channel-forming proteins, new lines of investigation have been opened with which to investigate the potential role of these water channels in maintaining endolymph homeostasis and normal inner ear function. Recent reports intriguingly show that precise regulation of endolymph homeostasis is also relevant during development, with perturbation of endolymph regulation causing malformation of inner ear structures as well as deafness and vestibular dysfunction in humans and mouse models (Everett et al. 2001). These data indicate the need to examine the developmental expression and role of potential endolymph-regulating proteins in the inner ear.

At least ten mammalian aquaporins (AQP) have been identified (AQP0-9) and studied for their water-transporting functions in various organ systems. AQPs, for example, have been implicated in the regulation of fluid homeostasis as well as secretory functions in the kidney, lung, eye, and salivary glands (Neilsen et al. 1995; King et al. 1996; Knepper et al. 1997; Ma et al. 1999). AQPs are homotetrameric with each monomer containing six membrane-spanning $\alpha$ helical domains that form a distinct water pore. Highly conserved motifs within these monomers include two "NPA" sequences that are thought to play a role in forming the water pore (Borgnia et al. 1999). Both AQP1 and 5 structures allow for a bidirectional flow of water depending on osmotic pressure (Verkman et al. 2000).

Prior studies have examined the expression of AQP1 and 5 in different animal models of the adult inner ear but have not specifically addressed the ontogeny of these water channels from early otic development to postnatal stages. Stankovic et al. (1995) reported on the expression of AQP1 in mesenchymal cells and type III fibrocytes surrounding various regions of the guinea pig inner ear. Takumi et al. (1998) reported similar expression patterns of AQP1 in the rat while Mhatre et al. (1999) recently demonstrated the presence of AQP5 in the lateral wall of the cochlea, specifically in the external sulcus cells and cells of the spiral prominence of the apical turns of the cochlea. Beitz et al. (1999) used a reverse transcriptase-polymerase chain reaction (RT-PCR) method to identify AQP5 transcripts from organ of Corti and Reissner's membrane tissues of the rat inner ear. These data along with our ready access to highly specific, wellcharacterized antisera to AQP1 and 5, and, in particular, the availability of $A q p 5$ null mutant mice, prompted this examination of the expression of these AQPs in the developing mouse inner ear and the effects of loss of Aqp5 function in a mouse model. Our AQP1 and AQP5 expression data are generally consistent with the findings in other animal models but also show substantial differences in cochlear expression of AQP5 compared to earlier studies (Mhatre et al. 1999). Functional studies suggest that under normal (nonstress) conditions, loss of AQP5 function does not cause obvious hearing or balance dysfunction in mice nor any gross morphologic abnormalities of the inner ear in mice. This is notably in line with recent work by Li and Verkman (2001) who found that their AQP5 null mutants also demonstrate normal hearing when tested in a more limited frequency test range. Our data are discussed with reference to the current literature and the possible importance of water transport in inner ear fluid homeostasis.

\section{MATERIALS AND METHODS}

Tissue preparation

All animals were handled and maintained according to an IACUC and institutional approved protocol. 
FVB/N mouse embryos were harvested between E9.5 and E18.5. Matings were set up and the morning on which a vaginal plug was observed was designated embryonic day 0.5 . Somites were counted at the time of harvest to more accurately stage embryos. Postnatal day (P) FVB/N mice were also sacrificed at P2, P7, $\mathrm{P} 14, \mathrm{P} 28$, as well as 5-8-month-old Aqp5 knockout mice $\left(A q p 5^{-/-}\right)$and corresponding wild-type littermates $\left(A q p 5^{+/+}\right)$. The inner ears with surrounding tissue were processed for routine hematoxylin and eosin staining, ISH, and IHC as described below.

Harvested embryos and/or postnatal tissue were immediately fixed in $4 \%$ paraformaldehyde in $1 \times$ PBS $\mathrm{pH}$ 7.4. Samples P7 and younger were cryoprotected using a $30 \%$ sucrose solution following overnight fixation at $4^{\circ} \mathrm{C}$. After embedding in OCT (IMEB Inc., Chicago, IL), sections were cut in the coronal plane at 10-12 $\mu \mathrm{m}$ and collected on Superfrost Plus slides (Fisher Scientific International, Pittsburgh, PA). Sections were stored at $-80^{\circ} \mathrm{C}$ until use. For ages $\mathrm{P} 7$ and older, the specimen were decalcified in 0.1 M EDTA $\mathrm{pH} 7.6$ for 1-2 weeks at room temperature, dehydrated, and embedded in paraffin. Sections were cut in the coronal plane at 5-7 $\mu \mathrm{m}$, collected on Superfrost Plus slides, and stored at room temperature until use.

\section{In situ hybridization probe preparation}

The digoxigenin-labeled riboprobe for AQP1 was generated from a 431-bp PCR product corresponding to Mus musculus aquaporin 1 mRNA (GenBank Accession No. 6680709) nt. 813-1222. The sequence was amplified from mouse kidney cDNA using primers courtesy of Dr. Ann Akeson. This PCR product was then subcloned into a pGEM-T Easy vector (Promega, Madison, WI) with flanking T7 and Sp6 promotors to generate sense and antisense probes. The antisense riboprobe was synthesized using T7 RNA polymerase after Spel restriction digest, and the sense riboprobe was synthesized using Sp6 RNA polymerase after Ncol restriction digest.

Similarly, an 839-bp AQP5 sequence (nt. 484-1322 from Mus musculus aquaporin 5 mRNA, GenBank NM_009701.1) was used to generate sense and antisense probes for ISH extracted from a 4-1 lung mAQP5 cDNA clone.

\section{Antibodies}

A rabbit polyclonal antibody (\#4835-EXT) was generated against a synthetic peptide $C$ GQVEEYDLDAD DINSRVEMKPK-COOH corresponding to the last 22 amino acids of both the rat and the mouse AQP1 peptide sequence and conjugated to keyhole limpet hemocyanin (Zymed Laboratories, San Francisco, CA). The antibody was affinity purified using a sul- folink column from Pierce. A preimmune serum was IgG purified using an immobilized protein A column from Pierce Biotech, Rockford, IL. The antibody has been tested for immunoreactivity by immunoblot using lung and kidney tissue from both rat and mouse (Towne et al. 2000).

A rabbit polyclonal antibody (LL639) was generated against a synthetic peptide $C$ EPEEDWEDHREE RKKTIELTAH-COOH corresponding to the last 22 amino acids of both the rat and the mouse AQP5 peptide sequence and conjugated to keyhole limpet hemocyanin (Lofstrand Labs, Ltd, Gaithersburg, MD). The antibody was affinity purified using a sulfolink column from Pierce. A preimmune serum was IgG purified using an immobilized protein A column from Pierce. The antibody has been tested for immunoreactivity by immunoblot using lung tissue from both rat and mouse (Krane et al. 1999).

\section{In situ hybridization (ISH)}

The in situ hybridization experiments were performed using a standard protocol with minor modifications (Choo et al, 1998; Morsli et al. 1998). The tissue sections were postfixed and permeabilized using $10 \mu \mathrm{g} / \mathrm{ml}$ proteinase $\mathrm{K}$ for $2-4 \mathrm{~min}$. Hybridization was performed in heat-sealable bags overnight at $70^{\circ} \mathrm{C}$ with a probe concentration of $0.6 \mu \mathrm{g} / \mathrm{ml}$. Detection with an alkaline phosphatase reaction produced a blue reaction product as the localization signal. The appropriate controls were performed using digoxigenin-labeled sense probes (generated by transcribing in the reverse orientation to the antisense probe) as well as no probes.

\section{Immunohistochemistry (IHC)}

The immunohistological experiments used a standard 3,3-diaminobenzidine (DAB) protocol with minor modifications (Zimmer and Lehman 1994). After thawing, slides were rehydrated and washed in $0.1 \mathrm{M}$ phosphate buffer with $0.1 \%$ Triton X-100 (PBTX). Sections were bleached in $0.5 \% \mathrm{H}_{2} \mathrm{O}_{2}$ in methanol and then in $0.5 \% \mathrm{H}_{2} \mathrm{O}_{2}$ in PBTX in order to eliminate endogenous peroxidase activity. The slides were incubated in "preblock" (5\% goat serum in $0.1 \mathrm{M}$ PBTX) for $0.5-3 \mathrm{~h}$ at room temperature. Primary antibody incubations were performed for approximately $3 \mathrm{~h}$ at $4^{\circ} \mathrm{C}$ in the primary antibody diluted in preblock. With PBTX washes in between, the slides were incubated in $7.5 \mu \mathrm{g} / \mathrm{ml}$ biotinylated antirabbit antibody (Vector Labs, lnc., Burlingame, CA) diluted in PBTX and then Vectastain Elite ABC reagent diluted in PBTX. Using a DAB solution, slides were developed colorimetrically resulting in brown staining. The concentrations of the primary antibody were 

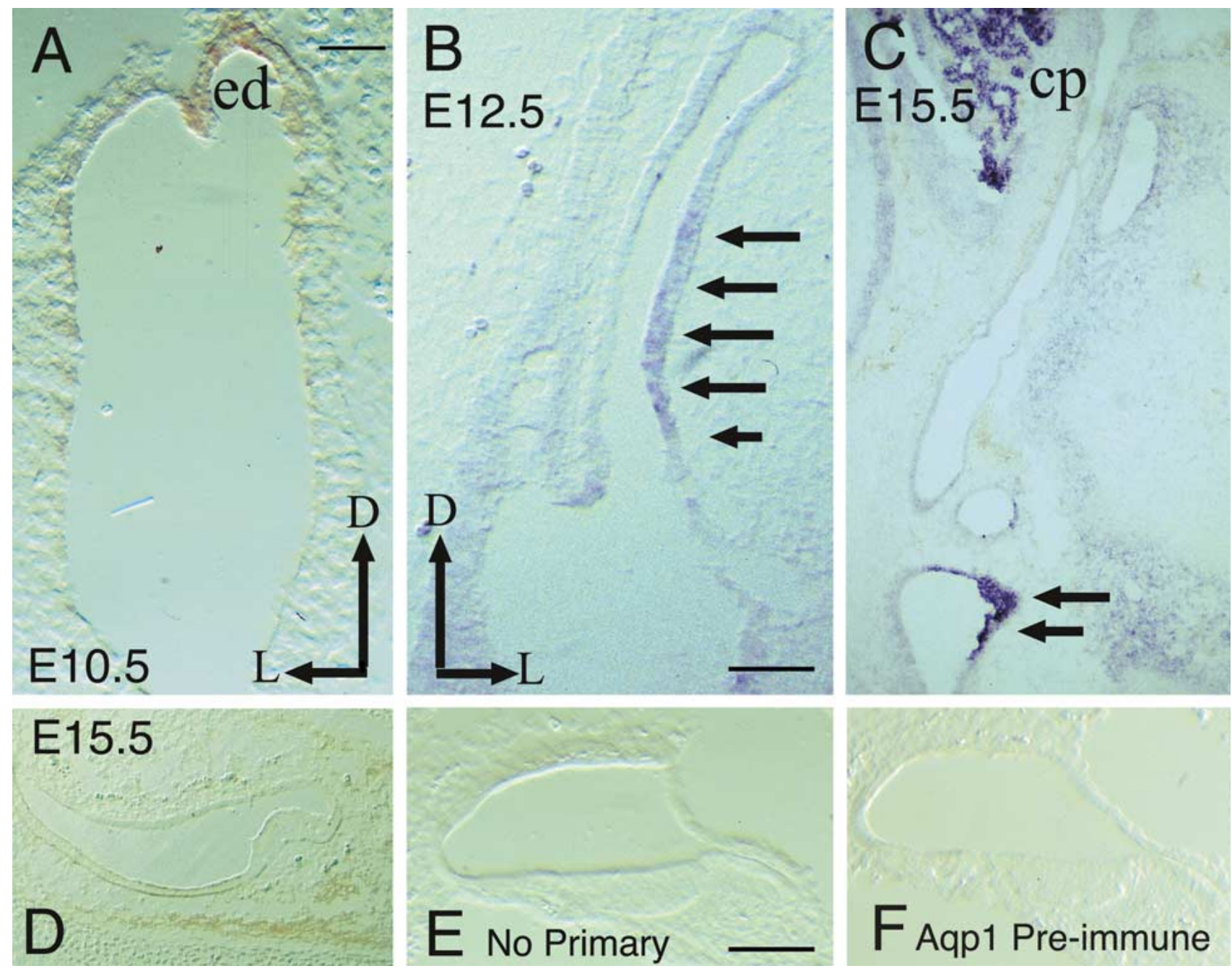

FIG. 1. IHC controls and AQP1 expression at E10.5-E15.5. A. IHC. AQP1 protein is expressed heterogeneously throughout the epithelium of the otocyst (E10.5) and particularly intensely in the forming endolymphatic duct. AQP1 transcripts are not seen until E12.5. B. ISH. At E12.5, AQP1 transcripts are expressed only in the lateral wallof the common crus (arrows) and vestibule. C. ISH. By E15.5, AQP1 mRNA is expressed in the lateral wall of the common crus, in the nonsensory portion of the saccule (arrows), and in the connective tissue surrounding the membranous labyrinth of the inner ear. It is also expressed in the choroid plexus. D. AQP1 protein expression is similar to AQP1 mRNA at E15.5 and immunostaining

as follows: $0.101 \mu \mathrm{g} / \mathrm{ml}$ anti-AQP1 antibody for the ages E10.5-E12.5, $0.076 \mu \mathrm{g} / \mathrm{ml}$ anti-AQP1 antibody for ages E15.5-P2, $0.148 \mu \mathrm{g} / \mathrm{ml}$ anti-AQP5 antibody for ages E10.5-P2, and $0.296 \mu \mathrm{g} / \mathrm{ml}$ anti-AQP5 antibody for ages $\mathrm{P} 7$ and older. The appropriate controls were done using preimmune serum as well as no primary antibody.

\section{Characterization of AQP5 Knockout mice}

The $A q p 5^{-/-}$mice were generated by blastocyst-mediated transgenesis and characterized by Krane et al. (2001). Routine behavioral, morphologic, and physi- surrounding the lateral ampulla is shown. (E, F). Representative cochlear sections from an E18.5 embryo. These late embryonic specimens were chosen because AQP1 is robustly expressed this developmental stage in these regions of the inner ear. The absence of any signal on these sections supports the specificity of the antisera used in this study. No primary antibody negative control (E) and AQP1 preimmune control (F). The axes in $\mathbf{A}$ apply to $\mathbf{D}, \mathbf{E}$, and $\mathbf{F}$, and axes in $\mathbf{B}$ also apply to $\mathbf{C}$. Scale bar in $\mathbf{A}=50 \mu \mathrm{m}$. Scale bar in $\mathbf{B}=100$ $\mu \mathrm{m}$ and applies to $\mathbf{C}-\mathbf{F}$. ed = endolymphatic duct; $\mathrm{cp}=$ choroid plexus.

ologic assessments were done through observation of appearance, presence or absence of circling-type behaviors, presence or absence of a Preyer's reflex, swim tests, histological studies, and formal audiologic assessment as described below.

\section{Auditory brainstem responses (ABR)}

Fourteen mice aged 5-8 months were tested for hearing loss using standard ABR techniques. Mice were anesthetized with avertin (25 mg/ $\mathrm{kg}$ body weight IP) and ABR measurements were performed in a quiet room. The mouse right ear was stimulated 


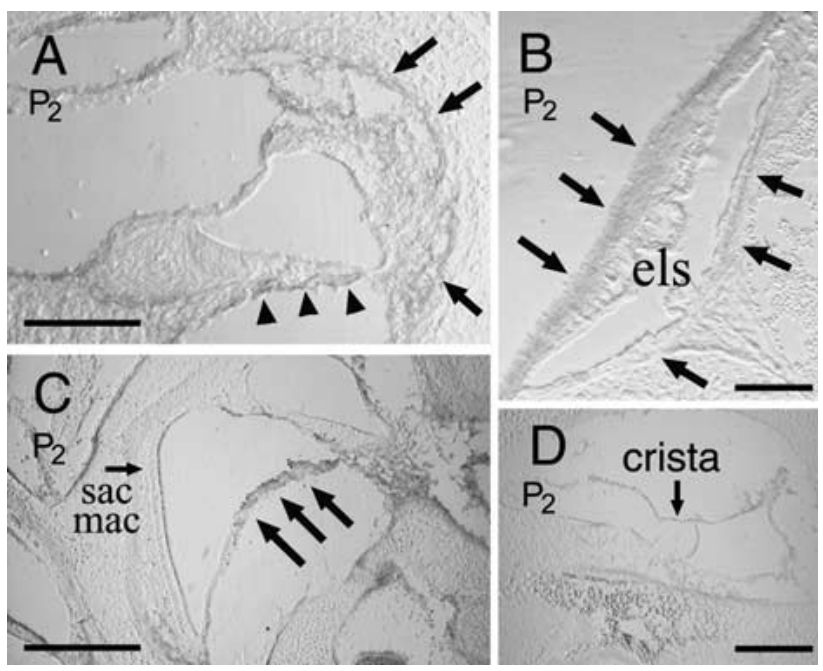

FIG. 2. Aquaporin 1 protein expression at postnatal day 2. A. AQP1 is expressed in the connective tissue surrounding the cochlea including the fibrocytes of the spiral ligament (arrows) and mesenchymal cells below the basilar membrane (arrowheads). It is not detected in the epithelial tissue. B. AQP1 protein is not detected in the epithelium of the endolymphatic sac but in a discrete layer immediately surrounding the epithelium. C. AQP1 is also expressed in the connective tissue surrounding the vestibular portion of the inner ear and robustly in nonsensory portion of the saccule (large arrows in C) and in the epithelium of the lateral wall of the common crus (not shown). The small arrow labels the macula of the saccule. D. Lateral ampulla. Arrow labels the crista. Scale bars in A-D=100 $\mu \mathrm{m}$. els= endolymphatic sac; sac mac $=$ saccule macula.

with a 0.1 -ms broadband click or a 3-ms tone burst of 8,16 , and $32 \mathrm{kHz}$ via a high-frequency transducer (Intelligent Hearing Systems, Inc., Miami, FL). ABRs were recorded using percutaneous needle electrodes (mastoid negative, vertex positive). The threshold of hearing was determined by observing the lowest-level intensity of sound required to elicit a characteristic waveform.

\section{RESULTS}

\section{AQP1 and AQP5 expression patterns}

Within the developing mouse inner ear, AQP1 protein was first detected heterogeneously throughout the epithelium of the otocyst (E10.5), with areas of more intense signal in the endolymphatic duct (Fig. 1A). AQP1 transcript was first detected at E12.5 in the lateral wall of the vestibule and common crus (arrows in Fig. 1B). At E12.5, AQP1 protein is expressed not only in the lateral wall of the common crus and vestibule, but heterogeneously throughout the epithelium of the membranous labyrinth and surrounding tissue of the endolymphatic duct and sac (data not shown). At later stages of development (E15.5-P2), AQP1 transcript is strongly expressed in the lateral wall of the common crus, the nonsensory portion of the saccule (arrows in Fig. 1C), and in the connective tissue surrounding the membranous labyrinth (Fig. 1C). AQP1 protein showed similar expression domains as the mRNA, but is more intensely expressed in the connective tissue surrounding the membranous labyrinth of the inner ear (E15.5-P2) (Figs. 1D and 2). AQP1 is also robustly expressed in the choroid plexus (Nielsen et al. 1993) and was used as an internal positive control (Fig. 1C). Negative controls for the in situ hybridization (sense probes) and immunohistochemistry (no primary antibody, preimmune serum) studies did not show any staining within the inner ear (Fig. 1E, F). Control data for AQP1 immunohistochemistry (Fig. 1E, F) are from E18.5 cochlear sections. These specimens were chosen since AQP1 is typically expressed robustly at this developmental timepoint in these tissues. The absence of any signal in these sections supports the specificity of the antisera used in this study.

Distinct signal for AQP5 transcript was first detected at E15.5 in the lateral cochlear duct epithelium in all turns of the cochlear duct (arrows in Figs. 3D and $4 \mathrm{~A}$ ). Figure $4 \mathrm{~A}$ and $\mathrm{A}^{\prime}$ show adjacent sections of the proximal E15.5 cochlea with arrows indicating the lateral epithelium. This pattern persists until E18.5, at which point signal is then restricted to a subset of cells on the lateral wall of the cochlea near the spiral prominence and is particularly intense in the more basal turns (arrows in Fig. 4B). This expression domain of AQP5 is observed through P2. AQP5 protein has a similar expression pattern as AQP5 mRNA (Fig.

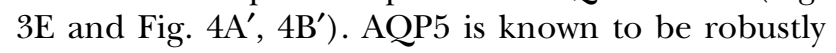
expressed in the salivary glands (Ma et al. 1999) and was used as a positive control for in situ hybridization experiments (Fig. 3B). Additionally, a very weak patch of AQP5 signal was noted in the presumptive interdental cell region at E18.5 by IHC but was not noted on ISH.

At P7, AQP5 protein is then expressed in the type II fibrocytes (Spicer and Schulte 1996) surrounding the spiral prominence in all turns of the cochlea (Fig. 5A). Significantly, the AQP5 expression pattern changed again by P14 (data not shown). Cochlear sections no longer show signal in the spiral prominence region but now show strong expression in the stria vascularis (Fig. 5B) that persists through adulthood.

\section{The inner ear in aquaporin 5 null mutant mice}

Generation and genotypic characterization of the Aqp5 mutant mouse strain has been described by Krane et al. (2001). An analysis of $A q p 5^{-/-}$mice (and control littermates) demonstrated no evidence of inner ear phenotype. Loss of AQP5 expression in the inner ear was confirmed by the absence of immuno- 

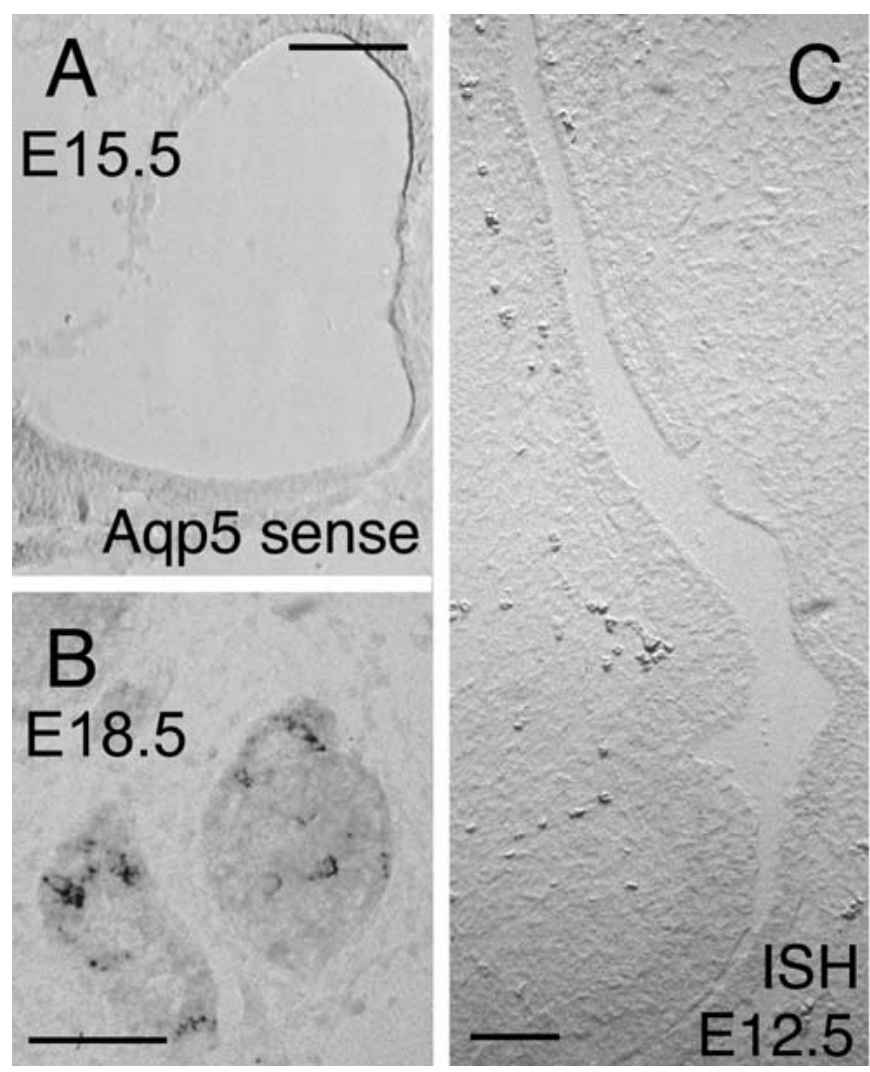

FIG. 3. Aquaporin 5 controls and early expression patterns. A. No signal was detected in control experiments with an AQP5 sense probe performed on E15.5 cochlear sections. B. ISH. AQP5 E18.5 mouse salivary gland. AQP5 has been reported to be expressed in mouse salivary glands and accordingly was used as a positive control (Krane et al. 1999). From E10 (15 somites, data not shown) through

staining in our cochlear sections by an antiaquaporin 5 antisera (Fig. 5C).

In general, AQP5 knockout mice averaged approximately $90 \%$ body weight of their littermates $\left(A q p 5^{+/+}\right.$and $\left.A q p 5^{+/-}\right)$with moderately reduced viability (as indicated by reduced birth rates of homozygous mutants) (Krane et al. 2001). Behaviorally, adult $A q p 5^{-/-}$mice show no circling or waltzing behaviors and swim normally, suggesting grossly normal vestibular function. In addition, Aqp5 mutants at 3 months of age showed normal Preyer's reflex indicating grossly normal hearing. In order to more quantitatively assess hearing in these mice, formal ABR testing was performed on $7 A q p 5^{+/+}$and $7 A q p 5^{-/}$ - age- and sex-matched mice at 5-8 months of age. These data show that under normal conditions, Aqp5 mutant mice show the same hearing sensitivity to 8-, $16-$, and $32-\mathrm{kHz}$ tone burst stimuli as their wild-type littermates. Distinct ABR waveforms were obtainable in all mice down to a $30 \mathrm{~dB}$ stimulus level (Fig. 6), After undergoing ABR testing, the same $A q p 5^{-1-}$ mice (and controls) were sacrificed and the inner ears harvested for routine histologic analysis. Histology of
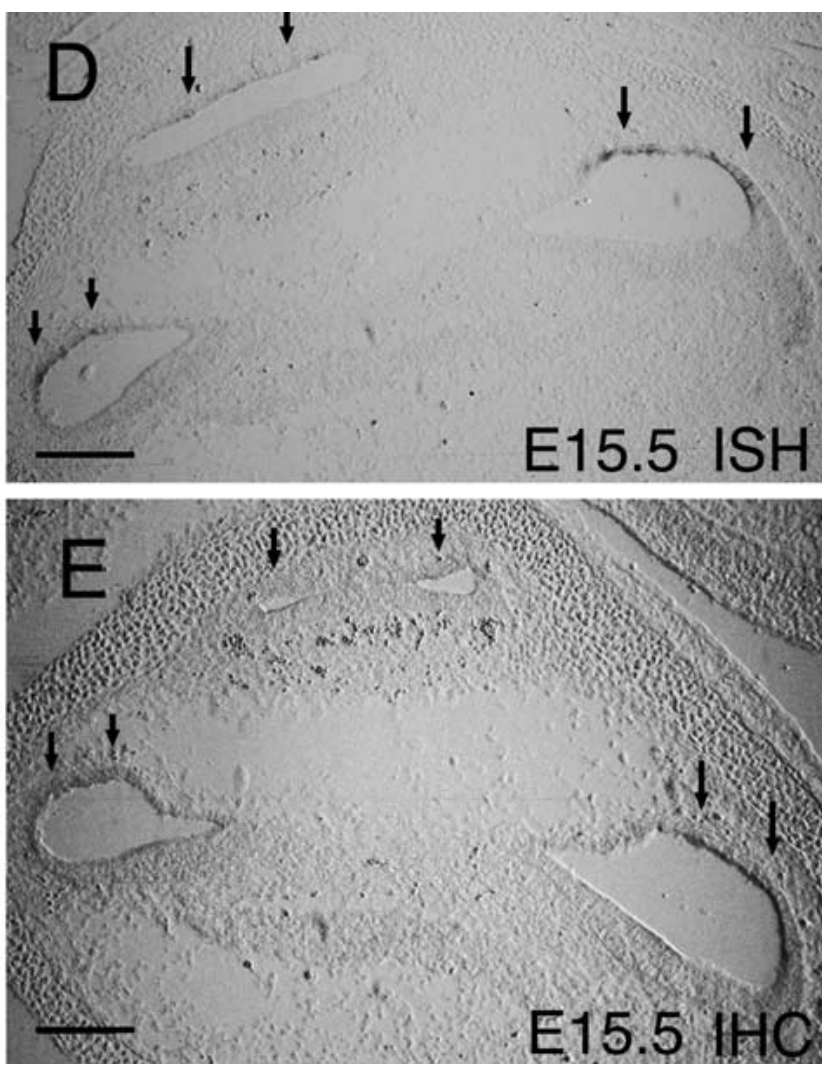

E15.0, AQP5 mRNA or protein is not detected. Sample negative in situ data from an E12.5 embryo is shown in C. Starting at E15.5, signals for AQP5 transcripts (arrows in D) and protein (arrows in E) were detected in the lateral wall of all turns of the cochlear duct (in a region corresponding to the presumptive stria vascularis) and were particularly intense in the basal turn. Scale bars in $\mathbf{A}-\mathbf{E}=100 \mu \mathrm{m}$.

$A q p 5^{-1-}$ inner ears showed no gross abnormalities (Fig. 5D). in particular, the endolymphatic and perilymphatic compartments showed no signs of dilatation or collapse, as might be postulated by a defect in fluid regulation.

\section{DISCUSSION}

The ontogenic expression of aquaporins 1 and 5

The examination of the temporal and spatial expression patterns of AQP1 and AQP5 highlights how two specific members of this family of water channelforming proteins exhibit unique and distinctive patterns of expression throughout development. AQP1, for example, is expressed significantly earlier than AQP5 and is initially noted in the epithelium of the otic vesicle. However, as the inner ear develops, AQP1 expression becomes more restricted to an extra-epithelial domain as well as a perilymph-bordering domain. By E15, AQP1 protein and transcripts are observed diffusely in the loose connective tissue cells surrounding the endolymphatic compartment and 


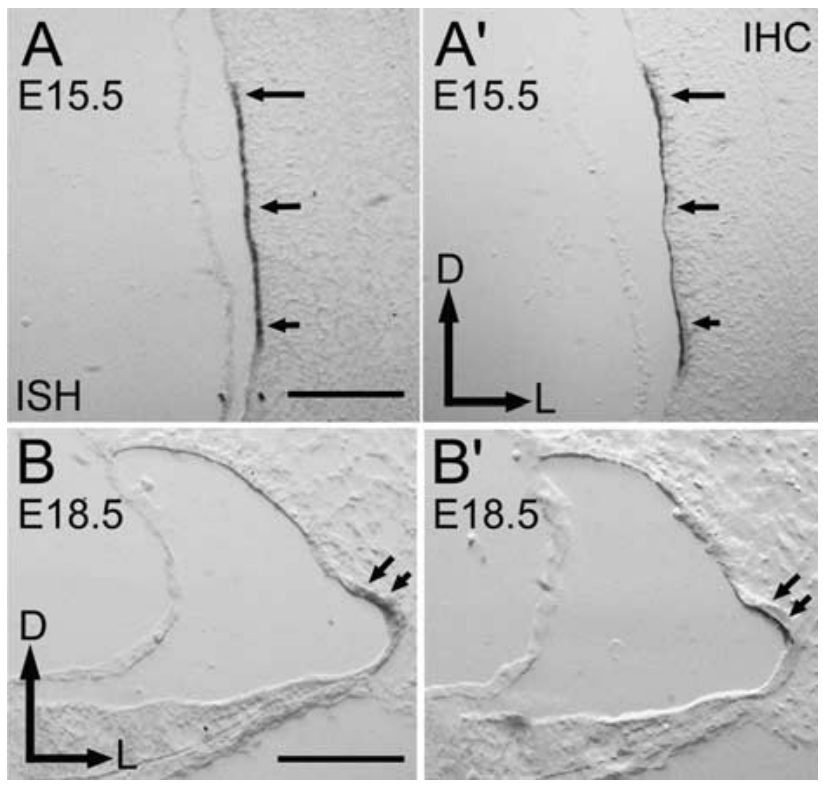

FIG. 4. AQP5 expression between E15.5 and E18.5. Comparison of the AQP5 signal in the lateral cochlear wall at E15.5 under higher magnification (arrows in $\mathbf{A}$ and adjacentsection $\mathbf{A}^{\prime}$ ) shows the transcript and protein signals to overlap without a demonstrable difference. AQP5 transcripts $(\mathbf{B})$ and protein $\left(\mathbf{B}^{\prime}\right)$ then restricted to a region inferior to the presumptive stria vascularis by E18.5 (arrows in $\mathbf{B}$ and $\mathbf{B}^{\prime}$ ) and remains in this domain through P2. Scale bars $=100 \mu \mathrm{m}$. Scale bar in $\mathbf{A}$ also applies to $\mathbf{A}^{\prime}$. Scale bar in $\mathbf{B}$ applies to $\mathbf{B}^{\prime}$.

bordering the perilymphatic spaces (e.g., the scala tympani, see Fig. 2A). Accordingly, its role may be related to the transport of water molecules external to the endolymphatic compartment and perhaps in regulating the osmotic environment immediately adjacent to the scala media. Also possible is a specific role for AQP1 in water transport into or out of the perilymph depending upon osmotic conditions. The expression of AQP1 in the mesenchymal cells underlying the basilar membrane and in type III and IV fibrocytes (Spicer and Schulte 1991) that contact the perilymphatic compartment supports this hypothesis. Previous studies on adult guinea pigs (Stankovic et al. 1995) and rats (Takumi et al. 1998) have also described AQP1 signal in type III fibrocytes of the spiral ligament and have suggested a possible role in volume regulation.

An exception to this extra-epithelial expression pattern is the particularly intense and focal expression of AQP1 in the nonsensory portion of the saccule. The function of AQP1 in this region of the inner ear is not clear. It is also not clear why AQP1 expression would be restricted to one otolilthic organ (the saccule) and not the other (the utricle). This observation led us to examine the differences between these two related organs in an attempt to explain the differential expression patterns we observed. At a morphological level, the utricle and
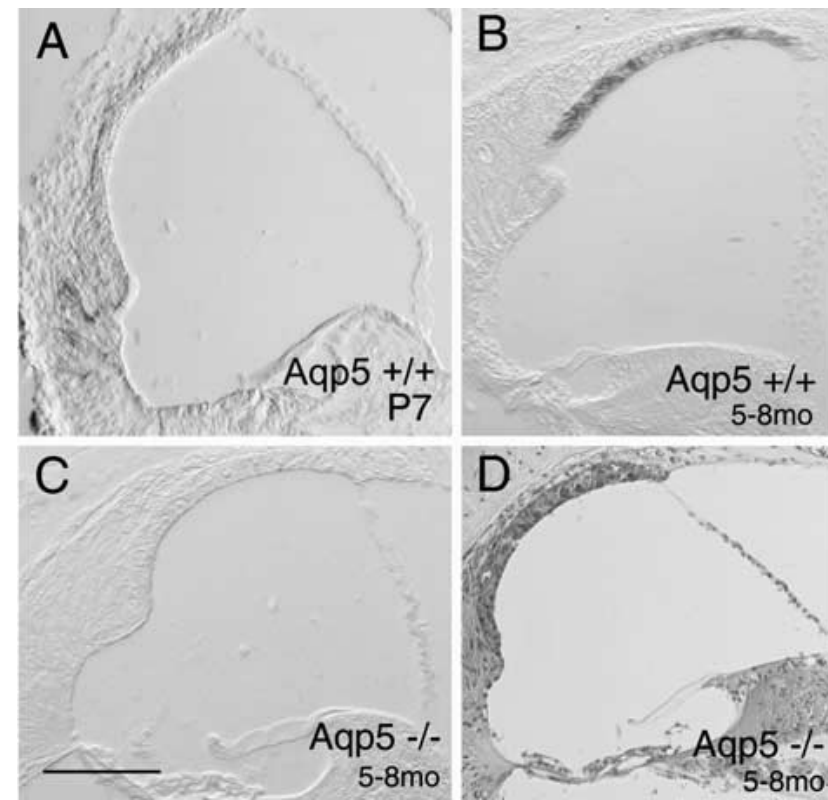

FIG. 5. Postnatal AQP5 expression and histology of aquaporin 5 deficient mice. A. At P7, AQP5 protein was detected in the type II fibrocytes surrounding the spiral prominence. By P14, the expression pattern of AQP5 protein again shifted from the spiral prominence region to the stria vascularis. The more apical turns continued to show signal in the cells near the spiral prominence (not shown), while the basal turns demonstrated signal only in the stria vascularis. At 4 weeks (not shown) and B. 5-8 months, AQP5 protein is expressed only in the stria vascularis. C. IHC. No anti-AQP5 staining was demonstrable in AQP5 -/- inner ears at 5-8 months of age. D. AQP5 -/- hematoxylin and eosin-stained tissue sections. Note the normal appearance of the cochlear duct with a well-preserved organ of Corti and normal-appearing Reissner's membrane. Areas of normal AQP5 expression (along the lateral cochlear wall) similarly appear unaffected compared with controls. Scale bar in $\mathbf{A}=100 \mu \mathrm{m}$ and appliesto B, C, and $\mathbf{D}$.

saccule are similar in possessing a macula (a sensory hair cell region) and otoliths that are believed to provide an inertial mass that helps these organs detect linear acceleration. However, these organs differ in that the utricle shows a dark cell population (comparable to the dark cells of the ampullae) that is believed to be involved in endolymph generation (Kimura et al. 1963). This suggests that the utricle may have an endolymph secretory role while the saccule does not. Consistent with this theory are the findings by Fina and Ryan (1994) which showed that utricular dark cells robustly express transcripts for the $\alpha 1$ and $\beta 2$ subunits of $\mathrm{Na}, \mathrm{K}-\mathrm{ATPase}$, an isoform combination associated with transport of sodium against high gradients (as in the stria vascularis). The absence of dark cells and this $\mathrm{Na}, \mathrm{K}-\mathrm{ATPase}$ isoform expression pattern in the saccule also suggests that the saccule is not intimately involved in endolymph generation. Taken together, AQP1 is unlikely to be involved in an endolmyph secretory role in the saccule (since the saccule does not appear to be involved 
A

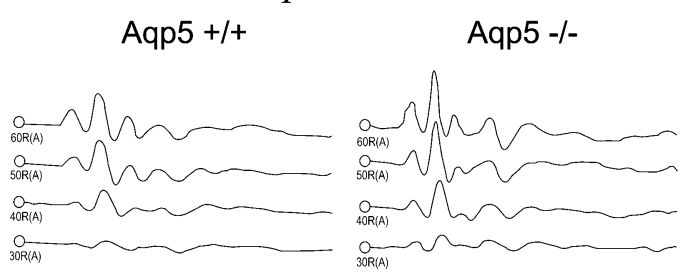

B

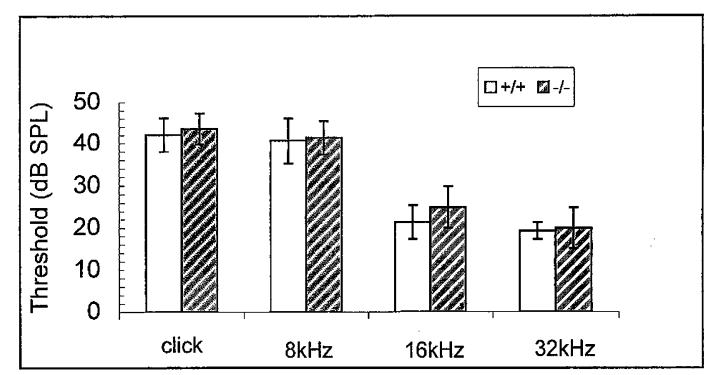

Error bars +/- 1 S.D.

FIG. 6. Auditory brainstem responses (ABR). A. Representative ABR recordings from $A q p 5^{+/+}$(left) and $A q p 5^{-/-}$(right) mice exposed to a broadband click in the 40-70 dB SPL range. Both Aqp $5^{+/+}$and Aqp $5^{-1-}$ mice showed observable responses to click sounds of $40 \mathrm{~dB}$. B. Summary of ABR thresholds for Aqp5 $5^{+/+}$(open bars, $n=6$ ) and Aqp $5^{-1-}$ (hatched bars, $n=6$ ) mice in response to the clicks and the three pure tones at 8,16 , and $32 \mathrm{kHz}$. The difference in mean $A B R$ thresholds between $A q p 5^{+/+}$and Aqp $5^{-/-}$mice was not statistically significant. Values shown are mean \pm standard deviation.

in this process in the mouse inner ear). We can only speculate that the robust expression of AQP1 in the saccule during development of the mouse inner ear facilitates rapid fluxes of water either into or out of the endolymphatic compartment as the inner ear undergoes a dramatic morphogenesis. Further work is also continuing in an attempt to identify other potential AQPs that may be expressed in the utricle and that might play a role in endolymph production.

AQP5 shows a markedly different developmental expression pattern in the mouse inner ear. First noted at E15.5 in the lateral wall of the cochlear duct, by E18.5 the AQP5 expression domain is further restricted to an epithelial region just inferior to the stria vascularis and centered around the spiral vessel. This precise expression pattern is consistent with earlier reports on the expression of AQP5 protein in the rat inner ear (although subtle differences are discussed below). However, the striking features of AQP5 developmental expression are the highly specific and variable domains of expression before and after birth. Initially, AQP5 was detected at E15.5 in an area that is the presumptive stria vascularis (see Figs. 3D,E and

$\left.4 \mathrm{~A}, \mathrm{~A}^{\prime}\right)$. This pattern of expression continues until E18.5 at which point AQP5 transcripts and protein become restricted to an area just inferior to the stria vascularis and spiral prominence (see Fig. 4B, $\mathrm{B}^{\prime}$ ). The expression of AQP5 then shifts to the type II fibrocytes surrounding the spiral vessel at P7 (Fig. 5A) and away from the lumenal epithelium. Several days later (P14), the expression domain of AQP5 then returns to the stria vascularis and remains there at least until 8 months of age (oldest specimen examined). Such a dynamically changing expression pattern suggests that the water-transporting function of AQP5 is precisely regulated and, therefore, presumably relevant to fluid homeostasis in the inner ear. We speculate that such varying expression domains of AQP5 may be needed in precise regions to move water as these specific functional areas of the inner ear mature during development.

\section{AQP-mediated water transport in the} developing lateral cochlear wall

The lateral wall of the cochlear duct (and the stria vascularis, specifically) has long been proposed to play a major role in endolymph secretion and homeostasis (Lawrence 1991; Spicer and Schulte 1996). However, our AQP data and that from earlier reports show that the lateral cochlear wall can be further broken down into discrete cell populations that at a molecular level appear to have distinct functions (Fig. 7). Interestingly, the expression of AQP5 seemingly "migrates" between functional subdomains of the lateral cochlear wall at very precise timepoints preand postnatally.

Building upon the work of Everett et al. (2001), we utilized gene expression patterns as markers for the stria vascularis (Trp2), the external sulcus cells (BMP4), as well as chloride-iodide-transporting cells (Pendrin) in this lateral cochlear wall. With these markers as reference points, we have shown that at early postnatal periods (P2), the AQP5-expressing domain is composed of cells just inferior to the Trp2expressing domain yet lateral to the presumptive Hensen's and Claudius cells (as defined by their BMP4 expression) (Morsli et al. 1998). Furthermore, the AQP5-expressing cells can be defined as a subset of the cells expressing Pendrin, a putative anion transporter (Everett and Green 1999), along the lateral wall of the cochlear duct inferior to the stria vascularis. These data suggest a particularly significant water- and anion-transporting role concentrated in this specific region of the inner ear and, therefore, a likely significant role in endolymph homeostasis. However, postnatally, the expression of AQP5 continues to show marked changes with a shift of expression domain to the spiral prominence region at 

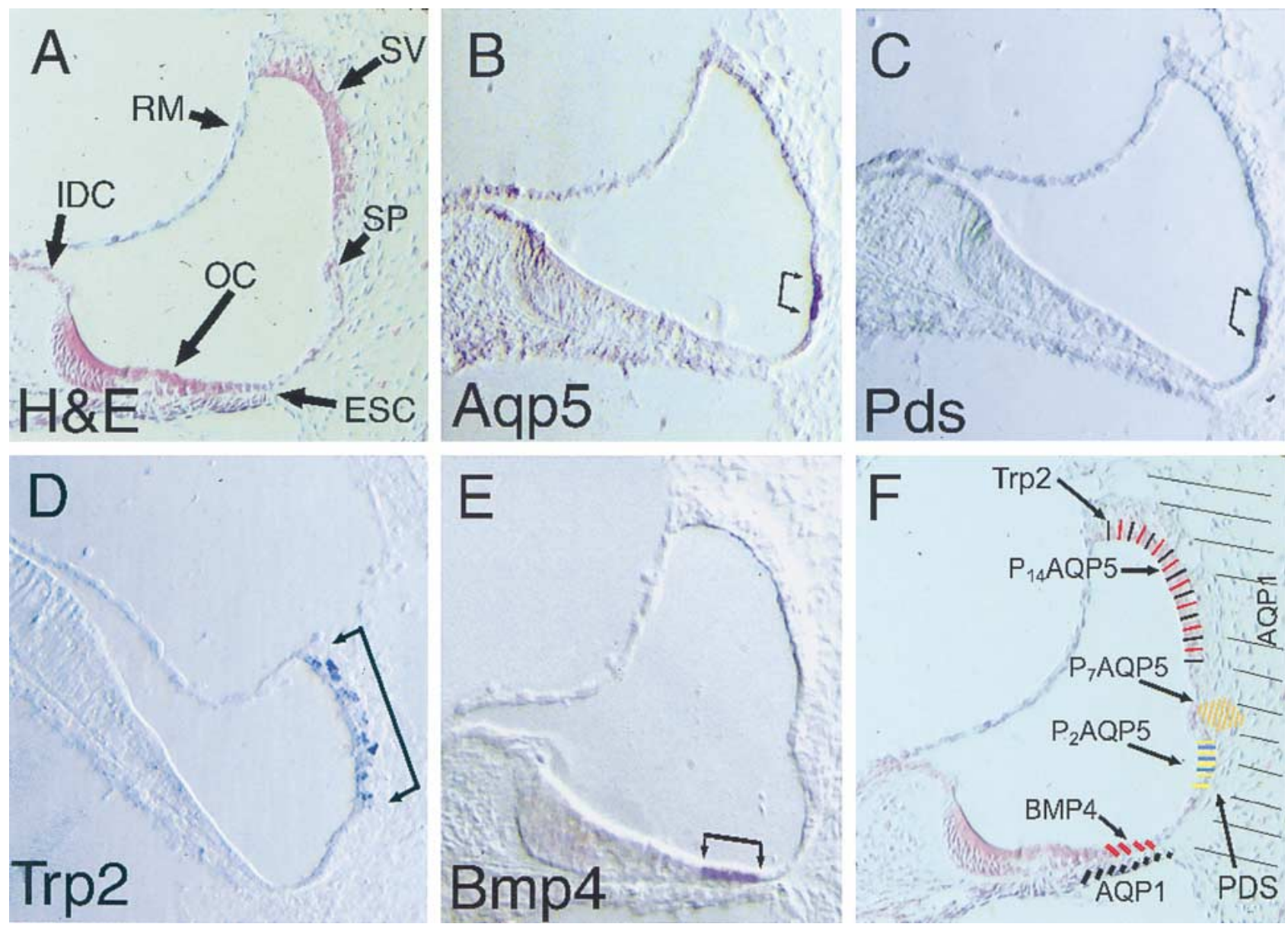

FIG. 7. Postnatal functional differentiation of the lateral cochlear wall. A. HE cochlea. Building upon the work of Everett et al. (1999) and data reproduced in ourstudies, (B) P2 AQP5 expression localizes to a subset of cells expressing $(\mathbf{C})$ Pendrin (Pds) immediately inferior to the stria vascularis. D. Tyrosinase-related protein 2 (TRP-2) marks the melanocytes in the stria vascularis while (E) bone morphogenetic protein 4 (BMP4) was used to mark the presumptive Hensen and Claudius cells of the external sulcus. F. Schematic drawing of molecular markers. Hatchings along the epithelium of the cochlear duct indicatethe expression domain of each gene. Black crosshatchings along the SV, Trp2; red cross-hatchings along the SV, AQP5 at P14; orange cross-hatchings, AQP5 at P7; yellow cross-hatchings,

P7 and then to the stria vascularis by P14. Noteworthy, this is also an important functional maturation period for the mouse inner ear during which mouse auditory function is felt to reach near mature levels (Kikuchi and Hilding 1965; Mikaelian 1979; Sadanaga and Morimitsu 1995). The expression of genes such as NKCC1 (a sodium, potassium, chloride cotransporter) have also been noted to change significantly in the gerbil inner ear during this early postnatal timeframe (Sakaguchi et al. 1998). Interestingly, studies of monovalent ion concentrations and the endocochlear potential (EP) in the mouse cochlea at early postnatal timepoints (P1, 3, 5, 7, 10, and 14) show that $\mathrm{K}^{+}, \mathrm{Na}^{+}$, and $\mathrm{Cl}^{-}$gradients are already established by $\mathrm{P} 1$, although not at fully mature
PDS; blue cross-hatchings, AQP5 at P2; red cross-hatchings at the external sulcus, BMP4; black cross-hatchings along the inferior edge of the basilar membrane area and along the spiral ligament, AQP1. Although data from adjacent sections stained with AQP1 are not included in this diagram, the hatchings along the inferior margin of the basilar membrane and along the fibrocytes of the spiral ligament are labeled to show the relative domains of AQP1 expression. PDS, pendrin; Trp2, tyrosinase-related protein 2; BMP4, bone morphogenetic protein 4. (Probe for PDS kindly provided by $\mathrm{L}$. Everett; Trp2 probe provided by K. Steel; BMP4 probe courtesy of D. $\mathrm{Wu}$ ). $\mathrm{OC}=$ organ of Corti; $\mathrm{RM}=$ Reissner's membrane; $\mathrm{SP}=$ spiral prominence; $\mathrm{SV}=$ stria vascularis.

levels until P14 (Yamasaki et al. 2000). Taking these observations together, we postulate that these changes in AQP5 expression domain accompany increased activity in these regions with respect to generation of the cochlea's electrochemical gradient and endocochlear potential. It seems reasonable to speculate that if these regions of the developing cochlea activate more vigorous ion transport mechanisms and establish steeper electrochemical gradients, water fluxes need to be managed more rapidly as well. As a result, it is reasonable to consider that carefully regulated water transport (by AQP5 and others) in the developing mouse inner ear plays a role in the developmental maturation of endolymph, and subsequently, in normal audition. 
The AQP1 expression data can be interpreted as AQP1 providing the extra-epithelial water channels involved in key endolymph homeostatic processes such as potassium recycling. This is based upon reports suggesting a recycling pathway for $\mathrm{K}^{+}$ions (after transduction of auditory stimuli) that carries $\mathrm{K}^{+}$ions back to the interstitial fibrocytes of the spiral ligament which then ultimately pump $\mathrm{K}^{+}$back into the endolymph at the strial marginal cells (Steel 2002). The expression domain of AQP1 at late embryonic and postnatal periods places it in precisely those regions believed to be involved in such potassium recycling. Further work is now needed to determine what interaction or facilitation water channel expression has with regard to potassium recycling. However, since rapid replenishment of $\mathrm{K}^{+}$ions into the endolymph are essential for maintaining an endocochlear potential and normal inner ear function, it seems plausible to hypothesize that rapid water transfer (via water channels) would be a concomitant consideration in rapid $\mathrm{K}^{+}$recycling.

The expression domains of AQP1 are also observed in cells surrounding the perilymphatic compartments. As such, AQP1 may be involved in moving water into or out of this fluid space rather than playing a specific endolymph homeostatic function.

Another plausible role that aquaporins might play in inner ear development is moving water into the inner ear compartments as they undergo growth and elongation. AQP1, for example, is noted in the early endolymphatic duct projection (E10.5) at a time when that anlagen is rapidly expanding dorsally (and the rest of the otocyst is also growing markedly). Presumably, a concomitant effect of elongation and growth would be an increase in fluid volume of the otocyst compartment. Aquaporin water channels would provide a means for rapidly moving water into the developing inner ear as this morphogenesis occurs. A failure to normally regulate the developing inner ear fluids (via anion transport) has been shown to result in dysmorphogenesis of the inner ear [e.g., Pendrin null mutant, (Everett et al. 2001)]. Therefore, it seems reasonable to suggest that water fluxes also need to be handled correctly during inner ear development.

\section{Aquaporin 5 function in the inner ear}

Based upon their observation that AQP5 was expressed specifically in the apical turns of the adult rat cochlea as opposed to the basal turn, Mhatre et al. (1999) hypothesized that AQP5 was relevant to lowerfrequency hearing in accordance with the tonotopic organization of the cochlea. Our data in the developing and early postnatal mouse inner ear contrastingly show AQP5 transcripts and protein in all turns of the cochlea. Therefore, we could not postulate any frequency-specific role for AQP5 in the mouse inner ear. Consistent with this conclusion is our assessment of adult $A q p 5$-deficient $\left(A q p 5^{-/-}\right)$mice demonstrating that, under normal conditions, hearing sensitivity is normal at 8,16 , and $32 \mathrm{kHz}$ (representing the hearing spectrum of mice). Histologic examination of the inner ears of $A q p 5^{-/-}$mice also shows normal morphology compared with controls. As a result, it appears that normal AQP5 function is not required for normal otic development or auditory function. Other compensatory/redundant mechanisms are likely involved in maintaining proper water homeostasis in $A q p 5^{-/-}$inner ears.

Pertinent to this discussion, Li and Verkman (2001) recently performed ABR tests on mice carrying a targeted deletion (individually) of the Aqp1, $A q p 3, A q p 4$, and $A q p 5$ genes. Based on their data, only Aqp 4 null mutant mice showed a significant loss of hearing. These data provided the first indication that aquaporin water channels can play a role in hearing. However, it is relevant to note that testing was performed between 4 and $20 \mathrm{kHz}$ in these studies. Mice typically display a broader frequency sensitivity range extending up to $32 \mathrm{kHz}$ (compared with $20 \mathrm{kHz}$ in humans). Accordingly, it would be interesting to examine the higher-frequency hearing in other AQP null mutant mice in order to determine if a highfrequency deficit can be demonstrated in other AQPdeficient mice.

Other lines of investigation into the regulation of AQP5 expression and/or activity point towards potential inner ear functions of AQP5. Reports on salivary secretion in response to cholinomimetic agents (e.g., pilocarpine) in $A q p 5^{-1-}$ mice show a marked difference (reduction) compared with controls (Krane et al. 2001). AQP5 has been shown to be expressed robustly in salivary gland tissue (see positive control data in Fig. 3B). Taken together, it is plausible that cholinergic effects on salivary gland secretion are mediated (at least in part) by AQP5 activity. Extrapolating that data to the inner ear, it then raises the possibility that AQP5 in the inner ear could be acted upon by analogous cholinergic agonists. Intriguingly, a report by Rubio et al. (1994) suggests that systemic pilocarpine in guinea pigs alters endolymph secretion in the inner ear and glycoprotein deposition in the tectorial membrane. Future studies will be designed to test the hypothesis that AQP5 in the inner ear may function as a mediator of cholinergically mediated regulation of inner ear fluid environment.

Other studies have recently shown that AQP5 mRNA and protein expression is downregulated by TNF- $\alpha$ by means of a TNFR1 and NF-KB pathway (Towne et al. 2001). The implications of this regulation through an inflammatory cytokine pathway cre- 
ate a potential link between inflammation and edema in the inner ear. Such data lead us to consider a potential relevance of AQP5 physiology to inner ear inflammation as well as clinical processes such as inflammatory or allergic endolymphatic hydrops.

The absence of specific AQP1 or AQP5 expression in developing or mature sensory cells (i.e., hair cells) suggests that their functional roles do not include an immediate or direct role in hair cell transduction of stimuli. However, Belyantseva et al. (2000) have demonstrated that guinea pig and rat outer hair cells display a water volume flow $\left(J_{v}\right)$ across the plasma membrane that is inhibited by $\mathrm{HgCl}_{2}$ (a reported inhibitor of aquaporin-mediated water transport) (Schnitzer and Oh 1996; Belyantseva et al. 2000). In addition, Belyantseva et al. (2000) reported that a pan-aquaporin antipeptide antibody labeled the lateral plasma membrane of outer hair cells, a region in which electromotility of these hair cells is believed to be generated. Accordingly, these intriguing data by Belyantseva et al. (2000) (on aquaporins in general) and our data specifically on AQP1 and 5 suggest that further investigations of AQP function in the inner ear are indicated in order to better determine the functional roles of these water channels in normal hearing and balance function, as well as to gain further insight into the complex processes maintaining inner ear endolymph homeostasis.

\section{ACKNOWLEDGMENTS}

The authors thank Krystyn Bourne, Maureen Luehrmann, Jennifer Towne, Ann Akeson, Bradford Mallory, Felisa Thompson, Valentina Pilipenko, and Alisa Reece for their technical assistance and/or critical review of this manuscript. This work was supported in part by the NIH DC00193 (D.C.) and NIH DE138283 (A.G.M.).

\section{REFERENCES}

Arenberg I. Results of the first 300 consecutive endolymphatic system-mastoid shunts with valve implants for hydrops. Otolaryngol. Clin. North Am. 16:153-174, 1982.

Beitz E, Kumagami H, Krippeit-Drews P, Ruppersberg J, Schultz J. Expression pattern of aquaporin water channels in the inner ear of the rat. The molecular basis for a water regulation system in the endoylmphatic sac. Hear. Res. 132:76-84, 1999.

Belyantseva IA, Frolenkov GI, Wade JB, Mammano F, Kachar B. Water permeability of cochlear outer hair cells: characterization and relationship to electromotility. J. Neurosci. 20:8996-9003, 2000.

Borgnia MJ, Kozono D, Calamita G, Maloney PC, Agre P. Functional reconstitution and characterization of AqpZ, the E. coli water channel protein. J. Mol. Biol. 291:1169-1179, 1999.

Choo D, SAnne J, Wu D. The differential sensitivities of inner ear structures to retinoic acid during development. Dev. Biol. 204:136-150, 1998.
Claes J, Van de Heyning PH. A review of medical treatment for Meniere's disease. Acta Otolaryngol. Suppl. 544:34-39, 2000.

Corey D, Hudspeth A. Ionic basis for the receptor potential in a vertebrate hair cell. Nature 281:675-679, 1979.

Everett LA, Belyantseva IA, Noben-Trauth K, Cantos R, Chen A, Thakkar SI, Hoogstraten-Miller SL, Kachar B, Wu DK, Green ED. Targeted disruption of mouse Pds provides insight about the inner-ear defects encountered in Pendred syndrome. Hum. Mol. Genet. 10:153-161, 2001.

Everett La, Glaser B, Beck JC, Idol JR, Buchs A, Heyman M, Adawi E, Hazani E, Nassir E, Baxevanis AD, Sheffield VC, Green ED. Pendred syndrome is caused by mutations in a putative sulphate transporter gene (PDS). Nat. Genet. 17:411-422, 1997.

EVERETT LA, GREEN ED. A family of mammalian anion transporters and their involvement in human genetic diseases. Hum. Mol. Genet. 8:1883-1891, 1999.

Everett LA, Morsli H, Wu DK, Green ED. Expression pattern of the mouse ortholog of the Pendred's syndrome gene (Pds) suggests a key role for pendrin in the inner ear. Proc. Natl. Acad. Sci. USA 96:727-732, 1999.

Fina M, RyAn A. Expression of mRNAs encoding alpha and beta subunit isoforms of $\mathrm{Na}, \mathrm{K}$-ATPase in the vestibular labyrinth and endolymphatic sac of the rat. Mol. Cell. Neurosci. 5:604613, 1994.

Hudspeth A. The cellular basis of hearing: the biophysics of hair cells. Science 230:745-752, 1985.

Juhn S, Pearce J, Guzowski J. Sodium exchange time of perilymph. Arch. Otolaryngol. 212:213-214, 1976.

Karet FE, Finberg KE, Nelson RD, Nayir A, Mocan H, Sanjad SA, Rodrigulz-Soriano J, Santos F, Cremers CW, Di Pietro A, Hoffbrand Bi, Winiarski J, Bakkaloglu A, Ozen S, Dusunsel R, Goodyer P, Hulton SA, Wu DK, Skvorak AB, Morton CC, CuNNINGHAM MJ, JHA V, LifTON RP. Mutations in the gene encoding B1 subunit of H+-ATPase cause renal tubular acidosis with sensorineural deafness [see comments]. Nat. Genet. 21:84-90, 1999.

Kikuchi K, Hilding D. The development of the organ of Corti in the mouse. Acta Otolaryngol. 60:207-222, 1965.

Kimura R. Experimental pathogenesis of hydrops. Arch. Otolaryngol. 212:263-275, 1976.

Kimura RS, LundsQuist PG, Wersall J. Secretory epithelial linings in the ampullae of the guinea pig labyrinth. Acta Otolaryngol. 57:517-530, 1963.

King LS, Nielsen S, Agre P. Aquaporin-1 water channel protein in lung: ontogeny, steroid-induced expression, and distribution in rat. J. Clin. Invest. 97:2183-2191, 1996.

Knepper MA, Verbalis JG, Nielsen S. Role of aquaporins in water balance disorders. Curr. Opin. Nephrol. Hypertens. 6:367-371, 1997.

Krane CM, Fortner CN, Hand AR, McGraw DW, Lorenz JN, Wert Je, Towne Je, Paul RJ, Whitsett JA, Menon AG. Aquaporin 5-deficient mouse lungs are hyperresponsive to cholinergic stimulation. Proc. Natl. Acad. Sci. USA 98:14114-14119, 2001.

Krane CM, Melvin Je, Nguyen HV, Richardson L, Towne Je, DoeTSChMAn T, Menon AG. Salivary acinar cells from aquaporin 5deficient mice have decreased membrane water permeability and altered cell volume regulation. J. Biol. Chem. 276:2341323420, 2001.

Krane CM, Towne JE, Menon AG. Cloning and characterization of murine Aqp5: evidence for a conserved aquaporin gene cluster. Mamm. Genome 10:498-505, 1999.

Kubisch C, Schroeder BC, Friedrich T, Lutjohann B, El-Amraoui S, Marlin S, Petit C, Jentsch TJ. KCNQ4, a novel potassium channel expressed in sensory outer hair cells, is mutated in dominant deafness. Cell 96:437-446, 1999. 
LAWRENCE M. Introduction to inner ear (fluid) physiology. In: PAparella MM, Shumrick PA, Gluckman JL, MeyerhoffF WL (eds) Otolaryngology. W.B. Saunders, Philadelphia, 1991, pp 199-217 Li J, VERKMAN AS. Impaired hearing in mice lacking aquaporin-4 water channels. J. Biol. Chem. 276:31233-31237, 2001.

Ma T, Song Y, Gillespie A, Carlson EJ, Epstein CJ, Verkman AS. Defective secretion of saliva in transgenic mice lacking aquaporin-5 water channels. J. Biol. Chem. 274:20071-20074, 1999.

Mhatre A, Steinbach S, Hribar K, Hoque A, Lalwani A. Identification of aquaporin 5 (AQP5) within the cochlea: cDNA cloning and in situ localization. Biochem. Biophys. Res. Commun. 264:157-162, 1999.

Mikaelian DO. Development and degeneration of hearing in the C57/b16 mouse: relation of electrophysiologic responses from the round window and cochlear nucleus to cochlear anatomy and behavioral responses. Laryngoscope 89:1-15, 1979.

Morsli H, Choo D, Ryan A, Johnson R, Wu D. Development of the mouse inner ear and its sensory organs. J. Neurosci. 18:33273335, 1998.

Naftalin L, Harrison M. Circulation of labyrinthine fluids. J. Laryngol. 72:118-136, 1958.

Nielsen S, Pallone T, Smith BL, Christensen EI, Agre P, Mauns$\mathrm{BACH}$ AB. Aquaporin-1 water channels in short and long loop descending thin limbs and in descending vasa recta in rat kidney. Am. J. Physiol. 268 (6 Pt 2):F1023-F1037, 1995.

Nielsen S, Smith BL, Christensen EI, Agre P. Distribution of the aquaporin CHIP in secretory and resorptive epithelia and capillary endothelia. Proc. Natl. Acad. Sci. USA 90 (15):7275-7279, 1993.

Paparella M. Pathogenesis and pathophysiology of Meniere's disease. Acta Otolaryngol. Suppl. (Stockh.) 485:26-35, 1991.

Peake W, Sohmer H, Weiss T. Microelectrode recordings of intracochlear potentials. MIT Res. Lab. Electronics Q. Progr. Rep. 94:293-204, 1969.

Rubio Me, Rueda J, Prieto JJ, Merchan JA. Pilocarpine-induced changes in the saccharide composition of the tectorial membrane and interdental cells of the organ of Corti: a study with gold-labeled lectins. J. Histochem. Cytochem. 42:405-416, 1994.

SAdanaga M, Morimitsu T. Development of endocochlear potential and its negative component in mouse cochlea. Hear. Res. 89:155-161, 1995.

SAJJADi H, Paparella MM, Williams T. Endolymphatic sac enhancement surgery in elderly patients with Meniere's disease [In Process Citation]. Ear Nose Throat J. 77:975-982, 1998.
Sakaguchi N, Crouch JJ, Lytle C, Schulte BA. Na-K-Cl cotransporter expression in the developing and senescent gerbil cochlea. Hear. Res. 118:114-122, 1998.

Schnitzer JE, OH P. Aquaporin-1 in plasma membrane and caveolae provides mercury-sensitive water channels across lung endothelium. Am. J. Physiol. 270 (1 Pt 2):H416-H422, 1996.

Schuknecht HF. Disorders of Unknown or Multiple Causes. Pathology of the Ear. Lea \& Febiger, Philedelphia, 1993, pp 499553-553

ShaH DK, Kartush JM. Endolymphatic sac surgery in Meniere's disease. Otolaryngol. Clin. North Am. 30:1061-1074, 1997.

Spicer SS, Schulte BA. Differentiation of inner ear fibrocytes according to their ion transport related activity. Hear. Res. 56:5364, 1991.

SPICER SS, Schulte BA. The fine structure of spiral ligament cells relates to ion return to the stria and varies with place-frequency. Hear. Res. 100:80-100, 1996.

Stankovic KM, Adams JC, Brown D. Immunolocalization of aquaporin CHIP in the guinea pig inner ear. Am. J. Physiol. 269:C1450-C1456, 1995.

STEEL KP. The benefits of recycling. Science 285:1363-1366, 2002.

SteEl KP, KRos CJ. A genetic approach to understanding auditory function. Nat. Genet. 27:143-149, 2001.

Takumi Y, Nagelhus EA, Eidet J, Matsubara A, Usami S, Shinkawa S, Nielsen S, OtTersen OP. Select types of supporting cell in the inner ear express aquaporin-4 water channel protein. Eur. J. Neurosci. 10:3584-3595, 1998.

Towne Je, Harrod KS, Krane CM, Menon AG. Decreased expression of aquaporin (AQP) 1 and AQP5 in mouse lung after acute viral infection. Am. J. Respir. Cell Mol. Biol. 22:34-44, 2000.

Towne JE, Krane CM, Bachurski CJ, Menon AG. Tumor necrosis factor-alpha inhibits aquaporin 5 expression in mouse lung epithelial cells. J. Biol. Chem. 276:18657-18664, 2001.

Verkman AS, Matthay MA, Song Y. Aquaporin water channels and lung physiology. Am. J. Physiol. Lung Cell Mol. Physiol. 278:L867-L879, 2000.

Welling DB, Pasha R, Roth LJ, Barin K. The effect of endolymphatic sac excision in Meniere disease. Am. J. Otol. 17:278-282, 1996.

Yamasaki M, Komune S, Shimozono M, Matsuda K, Haruta A. Development of monovalent ions in the endolymph in mouse cochlea. ORL J. Otorhinolaryngol. Relat. Spec. 62:241-246, 2000.

Zimmer K, Lehman M. Bromodeoxyuridine as a label for fetal hypothalamic whole tissue and suspension grafts. Neurosci. Prot. 3:46-57, 1994. 